\title{
Conditions for interference versus facilitation during sequential sensorimotor adaptation.
}

\author{
Otmar Bock ${ }^{1}$, Stefan Schneider $^{1}$, and Jacob Bloomberg ${ }^{2}$ \\ ${ }^{1}$ Institute of Physiology, German Sport University, Köln, Germany and \\ ${ }^{2}$ NASA-Johnson Space Center, Houston, USA.
}

Corresponding author: Dr. Otmar Bock

Head, Institute of Physiology

German Sport University

50927 Köln, Germany

Tel. $+49-221-4982370$

Fax $+49-221-4973531$

e-mail Bock@hrz.dshs-koeln.de 


\section{Abstract}

We investigated how sensorimotor adaptation acquired during one experimental session will influence the adaptation in a subsequent session. The subjects' task was to track a visual target using a joystick-controlled cursor, while the relationship between joystick and cursor position was manipulated to introduce a sensorimotor discordance. Each subject participated in two sessions, separated by a pause of two minutes to one month duration. We found that adaptation is achieved within minutes, and persists in memory for at least a month with only a small decay (Exp. A). When the discordances administered in the two sessions were in mutual conflict, we found evidence for task interference (Exp. B). However, when the discordances were independent, we found facilitation rather than interference (Exp. C); the latter finding could not be explained by the use of an "easier" discordance in the second session (Exp. D). We conclude that interference is due to an incompatibility between task requirements, and not to a competition of tasks for short-term memory. We further conclude that the ability to adapt to a sensorimotor discordance can be improved by practicing with an unrelated discordance.

Key words: humans; sensorimotor integration; motor learning; sensorimotor adaptation; tracking 


\section{Introduction}

Numerous studies have shown that human subjects can adapt to sensorimotor discordance, produced e.g. by visual (Bock, 1992; Pine et al., 1996; Stratton, 1897) or mechanical perturbations (Lackner and Dizio, 1994; Shadmehr and Mussa-Ivaldi, 1994). When the exposure to discordance is terminated, the adapted state can be stored in sensorimotor memory, and reactivated at a later time if required. This has been documented by experiments where subjects were adapted to a discordance, and were re-tested in a second session using the same or a stronger discordance: It was found that subjects' performance at the onset of the second session was better than at the onset of the first (Brashers-Krug et al., 1995; Klapp et al., 1974; Krakauer et al., 1999; Lazar and van Laer, 1968; Welch et al., 1993), indicating that the adapted state was partially or fully retained across the interval between sessions.

In other experiments, subjects were exposed in the second session to the opposite discordance than in the first (Shadmehr and Brashers-Krug, 1997; Shadmehr and Holcomb, 1999). When the two sessions were scheduled less than five hours apart, initial performance in the second session was substantially worse than in the first. Furthermore a third session, administered one or several days later, yielded no evidence for a retention of the originally adapted state. Taken together, these findings were attributed to a mutual interference between two concurrent adapted states. No such interference was found, however, when the first two sessions were more than five hours apart: In this case, performance in the second session was similar to that of naive subjects, and the third session yielded full retention of the originally adapted state. The cited findings were obtained during adaptation to an external force field, but mutual interference was also documented during adaptation to a rotated-visual display (Krakauer et al., 1999). 
To explain the above findings, it has been argued that adaptation needs several hours after exposure to consolidate from a fragile representation in short-term memory to a stable one in long-term memory. When the second task is presented too early, it will compete with the first for the limited capacity of short-term memory, therefore disturb the fragile original memory traces and, in turn, be disturbed by them. However, when the second task is presented after the original consolidation is completed, it can be acquired without mutual interference. Both adapted states can thereafter co-exist in long-term memory, as shown by studies where subjects were repeatedly exposed to multiple discordances: After some training, subjects could switch between several adaptive states in a context-dependent fashion (Shelhamer et al., 1992; Welch et al., 1993), or combine them to quickly adapt to a composite discordance (Flanagan et al., 1999).

The present study was designed to further explore the interaction of successive discordances in sensorimotor adaptation. In the cited experiments, the second discordance was always incompatible with the first: What has been learned in the first session was counterproductive to success in the second, and had to be suppressed in order to achieve satisfactory performance. We wondered whether a second discordance which doesn't require such suppression would produce interference as well. If so, we would have support for the above view, that interference is related to the fragility of memory traces, before consolidation to long-term memory. If not, interference would better be described as competition between conflicting task requirements. 


\section{Methods}

The experimental setup is illustrated schematically in Fig. 1a. Subjects stood in front of a vertical projection screen $S$, viewing it through a tilted mirror $M$, such that it appeared in a horizontal plane $\mathrm{H}$ at waist level. A visual target (luminous dot of $2 \mathrm{~cm}$ diameter) moved smoothly across the screen; the horizontal and vertical components of motion were each the sum of 5 sinewaves $(0.04,0.08,0.1,0.16$, and $0.2 \mathrm{~Hz})$, with a relative phase of $90 \mathrm{deg}$ between components. Thus, the target trajectory was repetitive with a cycle length of $50 \mathrm{~s}$, but subjects were not aware of this periodicity; for them, the trajectory was essentially unpredictable.

In their preferred hand, subjects held a springless, low-friction joystick which controlled the movement of a cursor on the screen. Thus the perceived motion of target, cursor, and joystick all occurred in the same - horizontal - plane. The subjects' task was to track the target with the cursor as accurately as possible. To prevent fatigue, each experimental was subdivided into tracking episodes of $50 \mathrm{~s}$ duration, separated by rest breaks. Subjects were free to terminate the breaks whenever they felt ready, typically within a few seconds.

The joystick position was sampled every $52 \mathrm{~ms}$ with a resolution of $0.1 \mathrm{deg}$ in the lateral, and $0.14 \mathrm{deg}$ in the sagittal dimension. The recorded signal was mapped onto displayed cursor position either non-inverted (e.g., leftward or forward joystick movement yielded a leftward or forward cursor movement, respectively), inverted along one of the two axes, or inverted along both axes, depending on the experimental condition (see below). The data were stored to hard disk, and the root mean square tracking error of each tracking episode was calculated using 
$R M S E=\sqrt{\frac{\sum_{i=1}^{n}\left(\Delta x_{i}\right)^{2}+\sum_{i=1}^{n}\left(\Delta y_{i}\right)^{2}}{n}}$

where $\Delta \mathrm{x}_{\mathrm{i}}$ and $\Delta \mathrm{y}_{\mathrm{i}}$ are the lateral and forward distance between target and cursor in the i'th data sample, and $\mathrm{n}$ is the number of samples analyzed. The first $500 \mathrm{~ms}$ of each tracking. episode were discarded from analysis, to prevent an initial misplacement of the cursor from having a substantial effect on RMSE.

84 volunteers participated in our study. None of them had previous experience in our task, or was familiar with playing joystick games. Each subject participated in only one of our four experiments. The subjects were 19 to 43 years of age, had corrected-for-normal vision and exhibited no overt sensorimotor or other neurological problems. As an incentive, we announced that the participant who achieves the lowest RMS score will receive a small monetary reward. All subjects signed an informed consent form for this study, which was approved by the Ethics Committee of the German Sport University.

\section{Experiment A}

The purpose of Exp. A was to confirm the validity of our experimental procedure as an adaptation paradigm, and to quantify the period of time over which the adapted state can be retained. 20 subjects participated in two experimental sessions. The first session started with 10 episodes under normal cursor control, i.e., the cursor always moved in the same direction as the joystick. This "warm-up-period was followed by 30 episodes where cursor control was manipulated: For 10 subjects, cursor movement was left-right reversed with respect to joystick movement, and for the other 10 subjects it was up-down reversed. 
All subjects were tested again in a second session for another 10 tracking episodes, using the same discordance as in the first (i.e., left-right or up-down reversal). The pause between sessions was $8 \mathrm{~min}, 25 \mathrm{~min}, 1$ hour, 2 hours or 1 month, and two subjects were assigned to each of the ten discordance $\mathrm{x}$ pause length combinations.

\section{Results and Discussion}

Fig. 1 shows raw tracking data of a subject during a warm-up episode (1b), the first episode under left-right reversal (1c), and a late episode under left-right reversal (1d). Clearly, tracking performance was severely degraded when the reversal was introduced, but recovered after prolonged exposure. This observation is confirmed by the RMSE data in Fig. 2: The tracking error increased dramatically after discordance onset, and then gradually settled at about the warm-up level. It can be further noted in Fig. 2, that RMSE at the onset of the second session was not much higher than at the end of the first.

As a measure of between-subject variability, we calculated the mean RMSE of each subject across all warm-up episodes, yielding values between 1.93 and $2.94 \mathrm{~cm}$. Overall variability was calculated as standard deviation across warm-up episodes and subjects, yielding $0.92 \mathrm{~cm}$.

For a statistical analysis, we compared the first RMSE value after reversal in session 1 with the mean of the last three values in that session. An analysis of variance (ANOVA) was applied, using the within-factor Episode (levels: first, last three) and the between-factor Discordance (levels: left-right, up-down). There was a significant main effect of Episode ( $F$ $(1,18)=233.1, p<0.001)$, which confirms that subjects were able to adapt, but no significant effects of Discordance and its interaction, which suggests that the initial and final errors with 
In another ANOVA, we compared the last three RMSE values of session 1 with the first three of session 2, using the within-factor Episode (last three, first three) and the between-factor Pause ( $8 \mathrm{~min}, 25 \mathrm{~min}, 1 \mathrm{hr}, 2 \mathrm{hrs}, 1$ month). Only the Episode $\mathrm{x}$ Pause interaction was significant $(F=4.15, \mathrm{p}<0.05)$, due to a slight increase of RMSE after the longest pause: At the end of session 1 , the RMSE (mean $+/-$ s.d.) was $3.32+/-0.65 \mathrm{~cm}$; it remained virtually unchanged with $3.29+/-0.69 \mathrm{~cm}$ at the beginning of session 2 after pauses of up to 2 hours, but increased somewhat to $4.00+/-0.38 \mathrm{~cm}$ after a one month pause. Although this increase was statistically significant, the RMSE was still substantially lower than at discordance onset in session $1(10.78+/-2.55 \mathrm{~cm})$, which indicates that retention of the adapted state remained nearly complete even after an extended pause.

In conclusion, Exp. A documents that our paradigm is suitable for the study of sensorimotor adaptation, and that it yields long-lasting retention. This finding is in accordance with previous work, reporting partial or full retention of adapted behavior even across substantial intervals (Brashers-Krug et al., 1995; Klapp et al., 1974; Krakauer et al., 1999; Lackner and Lobovitz, 1977; Lazar and van Laer, 1968).

\section{Experiment B}

Our second experiment was designed to replicate the previously observed negative interference between successive adaptations, and to explore over which time intervals this interference occurs. As in Exp. A, the first session consisted of 10 warm-up episodes, followed by 30 episodes under left-right or up-down reversal. The second session was administerēed after a pause of $2 \mathrm{~min}, 8 \mathrm{~min}, 25 \mathrm{~min}, 60 \mathrm{~min}, 2 \mathrm{hrs}, 18 \mathrm{hrs}$, 1 week or 1 month, and consisted of another 30 episodes under a different discordance: Subjects which have been 
and vice versa. A total of 32 subjects participated, two in each discordance $\mathrm{x}$ pause combination.

\section{Results and Discussion}

The RMSE values of one subject are plotted in Fig. 3. As in Exp. A, the error increased abruptly when the discordance was introduced in session 1, and then gradually decayed. At the onset of the second session, when the subject was confronted for the first time with a new type of discordance, the RMSE increased again, even to a distinctly higher level than in session 1 , and then decayed back to the baseline.

While the data in Fig. 3 are well fitted by an exponential function, data from a few subjects yielded no satisfactory fits with single and double exponentials, or other customary parametric functions. We therefore adopted the following alternative procedure to quantify the timecourse of all data sets. The initial error was determined as the first RMSE value under a discordance, the final error as the mean of the last three RMSE values in a session. We then calculated the half-time of decay by fitting a single exponential to all the remaining data points (i.e., to $30-4=26$ values), while at the same time forcing it to pass through the initial error, and to asymptotically approach the final error. We confirmed that the final error indeed represents the asymptote of decay by comparing the mean of episodes 38 to 40 with that of episodes 35 to 37 in a paired-samples t-test: The means were not significantly different ( $t(61)$ $=1,36 ; p>0.05$ ), which indicates that the decay was complete before session end. The values y.elded by this procedure are summarized in Table 1.

Each of the above three variables was subjected to an ANOVA, using the within-factor 
significant effect of Session is due to an increase of all three variable values from session 1 to 2 (see Table 1). The lack of a significant Session by Pause interaction indicates that we found no evidence for a dependence of any variable on pause length. This conclusion is supported by the outcome of a paired-samples t-test, yielding a significant difference between sessions even for the longest pause duration $(t=3.18, \mathrm{p}<0.05)$.

Our findings document that tracking performance in the second session is inferior to that in the first, which confirms previous data on interference between successive adaptations to noncompatible force fields (Shadmehr and Brashers-Krug, 1997; Shadmehr and Holcomb, 1999) and visual rotations (Krakauer et al., 1999). However, the available data disagree on the time scale of this effect: One group reported that interference is limited to pauses of up to 5 hours duration (Shadmehr and Brashers-Krug, 1997; Shadmehr and Holcomb, 1999), while the other group found interference even after 24-hours (Krakauer et al., 1999). Our own data are in accordance with the latter study, providing no evidence for a dependence on pause length for up to a full month. Thus, the slight decay of retention after one month pause observed in Exp. A was not reflected by a similar decay of interference in Exp. B.

The conflicting data on the time scale of interference in force-field studies and in the present work could be interpreted in at least two ways. Firstly, our subjects were exposed to their first discordance for 30 minutes, while the cumulated exposure time in the force field studies can be estimated as about eight minutes. It is possible that longer exposure produces more pronounced memory traces, which offer more resistance to conflicting task requirements. Secondly, dynamic tasks such as force field adaptation require the integrity of the lateral cerebellum, while mapping tasks such as a mirror-reversal don't (Fukuzawa et al., 1999), 
suggesting that adaptation to these discordances are based on different neuronal mechanisms. It wouldn't be surprising if different mechanisms had different interference characteristics ${ }^{1}$.

\section{Experiment C}

The purpose of this main experiment of our study was to introduce in the second session a discordance which is independent of the first. Thus, the two discordances should be neither conflicting as in Exp. B and in previous interference studies (Krakauer et al., 1999; Shadmehr and Brashers-Krug, 1997), nor synergic as in studies using incremental discordances of a single type (Lazar and van Laer, 1968; Welch et al., 1993). In our experiment C, the first session was similar to that of Exp. A and B, except that the warm-up period was reduced to five episodes in consideration of our subjects' patience. After a pause of $8 \mathrm{~min}, 2 \mathrm{hrs}, 1$ week or 1 month, the second session exposed all subjects to a $180-\mathrm{deg}$ rotation between joystick and cursor movement for 30 episodes. Note that this transformation represents a combination of left-right and up-down reversal, i.e., subjects which adapted to a left-right reversal in their first session now had to "add" an up-down reversal, and vice versa. 16 Subjects participated in Exp. C, two for each discordance x pause combination.

\section{Results and Discussion}

As expected, session 1 performance was similar to Exp. A and B, as illustrated by the data in Fig. 4 and Table 1. In contrast, the initial performance in session 2 was not worse than in session 1 , as expected in the case of negative interference, nor was it similar to session 1 , as it would be if both session were independent. Instead, initial performance in session 2 was 
substantially better than in session 1. The ANOVA results in Table 2 confirm that these changes were significant. The table further shows that the Session by Pause interaction was not significant, which suggests that the difference between session 1 and 2 persisted even after a one month pause.

In conclusion, the results of Exp. C seem to indicate that adaptation to one discordance can be beneficial for a subsequent adaptation to another discordance. This finding can not be explained by the argument that subjects have "partially" learned the second discordance by participating in the first session, as the two discordances were independent: Subjects adapting first tora left-right reversal have acquired no knowledge that would make a subsequent additional up-down reversal an easier transformation. Rather, it appears that by participating in the first session, subjects have improved their ability to adapt, and benefitted from this improvement in the second session. Such a phenomenon is called "learning to learn" in literature, but little experimental evidence supporting its existence has been presented in the past (see General Discussion).

However, an alternative interpretation of Exp. C is also conceivable. A 180-deg rotation might just be an "easy" type of discordance to adapt, irrespective of whether it is preceded by another session. Indeed, it has been shown that rotations of 180 deg are easier to learn than other rotational transformations (Cunningham, 1989). The following experiment was designed to scrutinize this possibility.

\section{Experiment D}

In this control experiment, the first session consisted of 35 episodes of warm-up (i.e., no 
Exp. C. Thus, subjects in this experiment had the same amount of experience with our apparatus as subjects in Exp. C, but were exposed to 180-deg rotation without prior exposure to another discordance. 16 Subjects participated, four for each pause duration.

Results and Discussion

The RMSE data of one subject are shown in Fig. 5, and are summarized across subjects in Table 1. It is quite obvious that the initial error in the second session of Exp. D was similar to the first session of Exp. C, but larger than in the second session of Exp. C. This observation was confirmed by ANOVAs (session 2 of Exp. D vs. session 1 of Exp. C: $F=0.25, p>0.05$; session 2 of Exp. D vs. session 2 of Exp. C: $F=6.44, p<0.05$ ). This outcome indicates that a $180 \mathrm{deg}$ rotation is not an easier adaptation task than a left-right or an up-down reversal, and thus rejects the alternative interpretation of Exp. C.

\section{General Discussion}

The present study dealt with adaptation to sensorimotor discordance administered in two successive sessions. We confirmed that the adapted state can remain in memory for at least a month (Exp. A), and that two non-compatible adapted states will interfere in memory even if they are acquired up to a month apart (Exp. B). However, when the two adapted states were compatible, we found facilitation rather than interference (Exp. C). The latter finding could not be explained by assuming that the second discordance was easier to learn (Exp. D).

It has been suggested in the past that interference during successive adaptations is due to the competition for short-term memory (STM) resources, and will subside once the originally 
shown a lack of interference between two tasks, one involving a kinematic, and the other a dynamic perturbation; this finding was not interpreted as a challenge to the above consolidation hypothesis, but rather as evidence for the existence of separate STM systems related to kinematic versus dynamic learning (Krakauer et al., 1999). However, the present findings are not as readily compatible with the consolidation interpretation, for two main reasons. Firstly, interference persisted in our study across exposure-free intervals of at least a full month, while consolidation should be completed within just a few hours (McGaugh, 1966). Secondly, we found interference only when the two tasks had conflicting, but not when they had independent requirements, while interference through consolidation should apply to any two tasks, as long as they concurrently require STM resources. Therefore, while consolidation might be a valuable concept for our understanding of motor learning, it can not explain interference in our study. In contrast, the other available interpretation, that interference is due to the conflict between two incompatible tasks (see Introduction), is in full agreement with the outcome of our Exp. B \& C.

Of course, the present findings do not imply that motor learning occurs without any involvement of the STM; we can only argue that limitations of STM storage space appear not to be the main reason of interference. However, other work provides experimental evidence that STM is indeed not a major factor in motor learning: It was found that electroconvulsive therapy may produce retrograde amnesia for sensorimotor activities while sparing the skills acquired through those very activities (Squire et al., 1984).

Probably the most interesting outcome of the present study is the facilitation when adapting to discordances which are independent, i.e., which have neither conflicting nor synergic task requirements (Exp. C). The very nature of independence precludes the interpretation, that 
the first. It rather appears that subjects have implemented some general strategies for coping with visuomotor transformations in the first session, and apply them to their benefit in the second session. If so, this phenomenon would reflect an improvement in the ability to adapt, called "learning to learn" in previous literature. However, previous evidence claiming support for this phenomenon has been equivocal at best: Subjects were exposed first to a small and then to a larger discordance of the same type, and it was found that adaptation in the second session was better than for control subjects who skipped the first (Lazar and van Laer, 1968; Welch et al., 1993). This finding probably doesn't reflect “learning to learn", but rather indicates that in the second session, subjects were able to build upon the knowledge they already have acquired by the end of the first. True "learning to learn" can only be confirmed in experiments where successive discordances are independent, as was the case in our Exp. C.

From our data, it appears that "learning to learn" is long-lasting, with beneficial effects even after an exposure-free interval of one full month. It would be interesting to determine the decay time of this phenomenon (if any), to find out whether it becomes even more expressed when more than two discordances are administered, and whether it generalizes to all adaptation tasks or rather is limited to similar discordance categories, as were the mirror reversals used in the present study. In particular, it has been shown that different categories of motor learning are based on different neural circuits (Fukuzawa et al., 1999; Ghilardi et al., 2000; Martin et al., 1996), and it is therefore quite conceivable that "learning to learn" is limited to tasks based in the same brain areas. Finally, it would be interesting to determine whether "learning to learn" and "interference" are two mutually exclusive phenomena, or whether they can occur concomitantly in the same task, with the one or the other phenomenon predominating, depending on specific task characteristics. 


\section{Figure legends}

Fig. 1. a: A schematic view of the experimental apparatus with back-projection screen (S), mirror(M), horizontal surface $(\mathrm{H})$, and joystick $(\mathrm{J})$; due to the mirror, the virtual screen position coincided with $\mathrm{H}$. b-d: Tracking performance of a subject before (a), immediately after (b) and 30 minutes after (c) introducing a left-right visuomotor reversal; the bold line represents target, and the thin line cursor movement.

Fig. 2. Tracking performance of one subject in Exp. A. Each dot represents the RMSE value for one 50-s tracking episode, and the curve indicates an exponential fit. The horizontal axis is interrupted where a pause occurred between session 1 and 2 .

Fig. 3. Tracking performance of one subject in Exp. B. For explanations see Fig. 2.

Fig. 4. Tracking performance of one subject in Exp. C. For explanations see Fig. 2.

Fig. 5. Tracking performance of one subject in Exp. D. For explanations see Fig. 2.

\section{Table legends}

Table 1. Means across subjects, and standard deviations, of the three response parameters determined in Exp. B-D.

Table 2. Summary of ANOVA results. Each line represents one analysis, with the dependent variable specified by the two leftmost columns. Data are F-values, and n.s., *, **, 


\section{Acknowledgments}

The present study was supported by the German Ministry for Education and Research (DLR grants 50 WB $9547 \&$ 9942); responsibility for the contents rests with the authors. Thanks are due to I. Benick for the development of experimental real-time software. Parts of the present work have been previously published in abstract form (2te Tagung Interdisziplinäre Bewegungsforschung, Saarbrücken, 1999; $5^{\text {th }}$ IBRO World Congress, Jerusalem, 1999; Soc. Neurosci. Abstr. 25, 2179, 1999). 


\section{References}

Bock O (1992) Adaption of aimed arm movements to sensorimotor disordance: evidence for direction-independent gain control. Behav Brain Res 51: 41-50

Brashers-Krug T, Shadmehr R, Todorov E. (1995) Catastrophic interference in human motor learning. In: Tesauro G, Touretzky DS, Leen TK (Eds.), Advances in neural information processing systems Vol. 7, Cambridge: MIT press, pp. 19-26

Cunningham HA (1989) Aiming Error Under Transformed Spatial Mappings Suggests a Structure for Visual - Motor Maps. J Exp Psychol: Human Percept Perform 15: 493 - 506

Flanagan JR, Nakano E, Imamizu H, Osu R, Yoshioka T, Kawato M (1999) Composition and Decomposition of Internal Models in Motor Learning under Altered Kinematic and Dynamic Enviroments. J Neurosci 19; RC34: 1-5

Fukuzawa K, Imamizu H, Nagai C, Iwata M, Kawato M (1999) Cerebellar Function Impairment In Tool-Use Learning: Different Internal Models For Rotational Transformation And Velocity Control. Soc Neurosci Abstr 25: 370

Ghilardi MF, Ghez C, Dhawan V, Moeller J, Mentis M, Nakamura T, Antonini A, Eidelberg D (2000) Patterns Of Regional Brain Activation Associated With Different Aspects Of Motor Learning. (manuscript under review)

Klapp ST, Nordell SA, Hoekenga KC, Patton CB (1974) Long - lasting aftereffect of brief 
Krakauer JW, Ghilardi M-F, Ghez C (1999) Independent learning of internal models for kinematic and dynamic control of reaching. Nature Neurosci 2: 1026-1031

Lackner JR, DiZio P (1994) Rapid Adaption to Coriolis Force Perturbations of Arm Trajectory. J Neurophysiol 72: 299-313

Lackner JR, Lobovitz D (1977) Adaptation to displaced vision: Evidence for prolonged aftereffects. Q J Exp Psychol 29: 65-69

Lazar G, van Laer J (1968) Adaptation to Displaced Vision After Experience with Lesser Displacements. Southern Universities Press 26: 579-582

Martin TA, Keating JG, Goodkin HP, Bastian AJ, Thach WT (1996) Throwing while looking through prisms. I. Focal olivocerebellar lesions impair adaption. Brain 119: 1183-1198

McGaugh JL (1966) Time-dependent processes in memory storage. Science 153: 1351-1358

Pine ZM, Krakauer JW, Gordon J, Ghez C (1996) Learning of scaling factors and reference axes for reaching movements. NeuroReport 7: 2357-2361

Shadmehr R, Brashers-Krug T (1997) Functional Stages in the Formation of Human LongTerm Motor Memory. J Neurosci 17: 409-419

Shadmehr R, Holcomb HH (1999) Inhibitory control of competing motor memories. Exp 
Shadmehr R, Mussa-Ivaldi FA (1994) Adaptive representation of dynamics during learning of a motor task. J Neurosci 14: 3208-3224

Shelhamer M, Robinson DA, Tan HS (1992) Context-specific adaptation of the gain of the vestibulo-ocular reflex in humans. J Vest Res 2: 89-96

Squire LR, Cohen NJ, Zouzounis JA (1984) Preserved memory in retrograde amnesia: sparing of a recently acquired skill. Neuropsychologia 22: 145-52

Stratton GM (1897) Vision without inversion of the retinal image. Psychol Rev 4: 341-481

Welch RB, Bridgeman B, Anand S, Browman KE (1993) Alternating prism exposure causes dual adaption and generalization to a novel displacement. Percept Psychophys 54: 195-204 


\section{Figures}

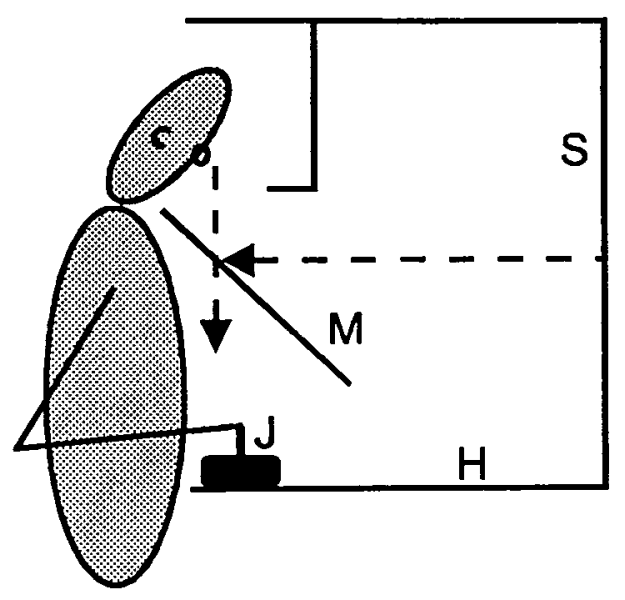

a)

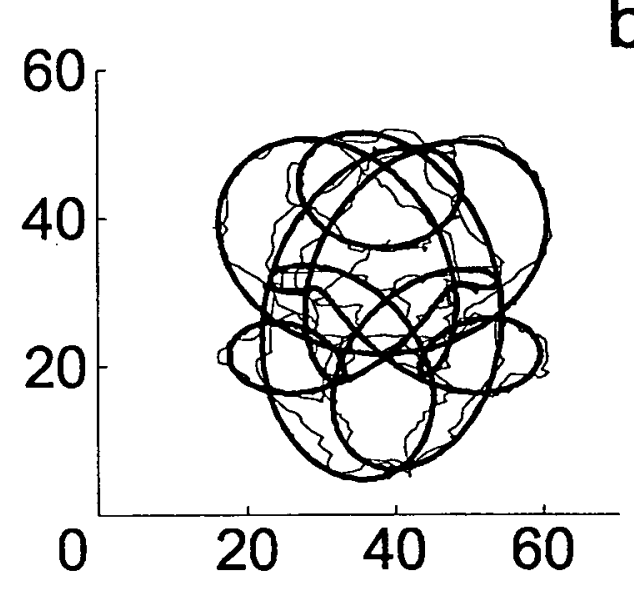

b)
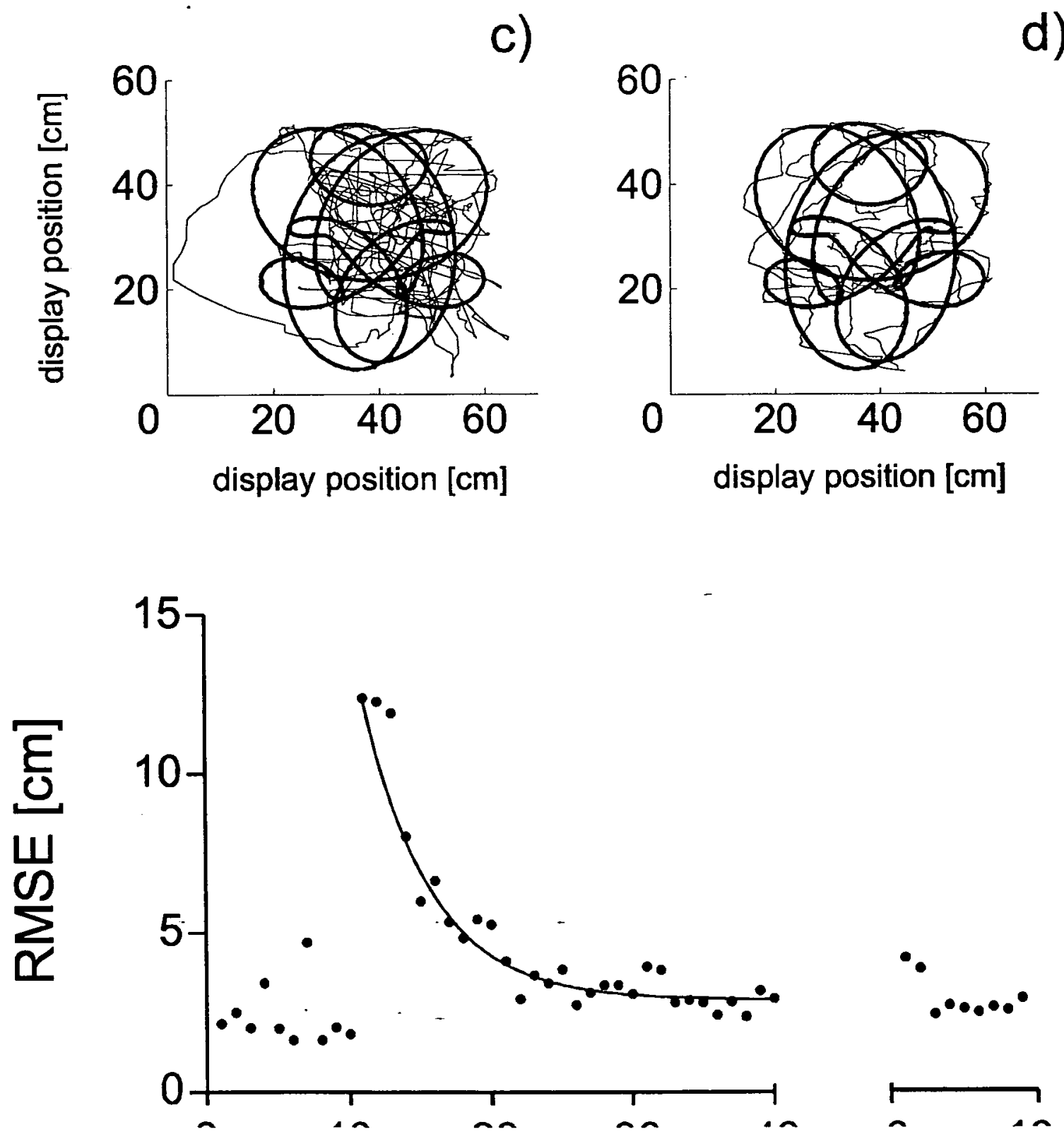

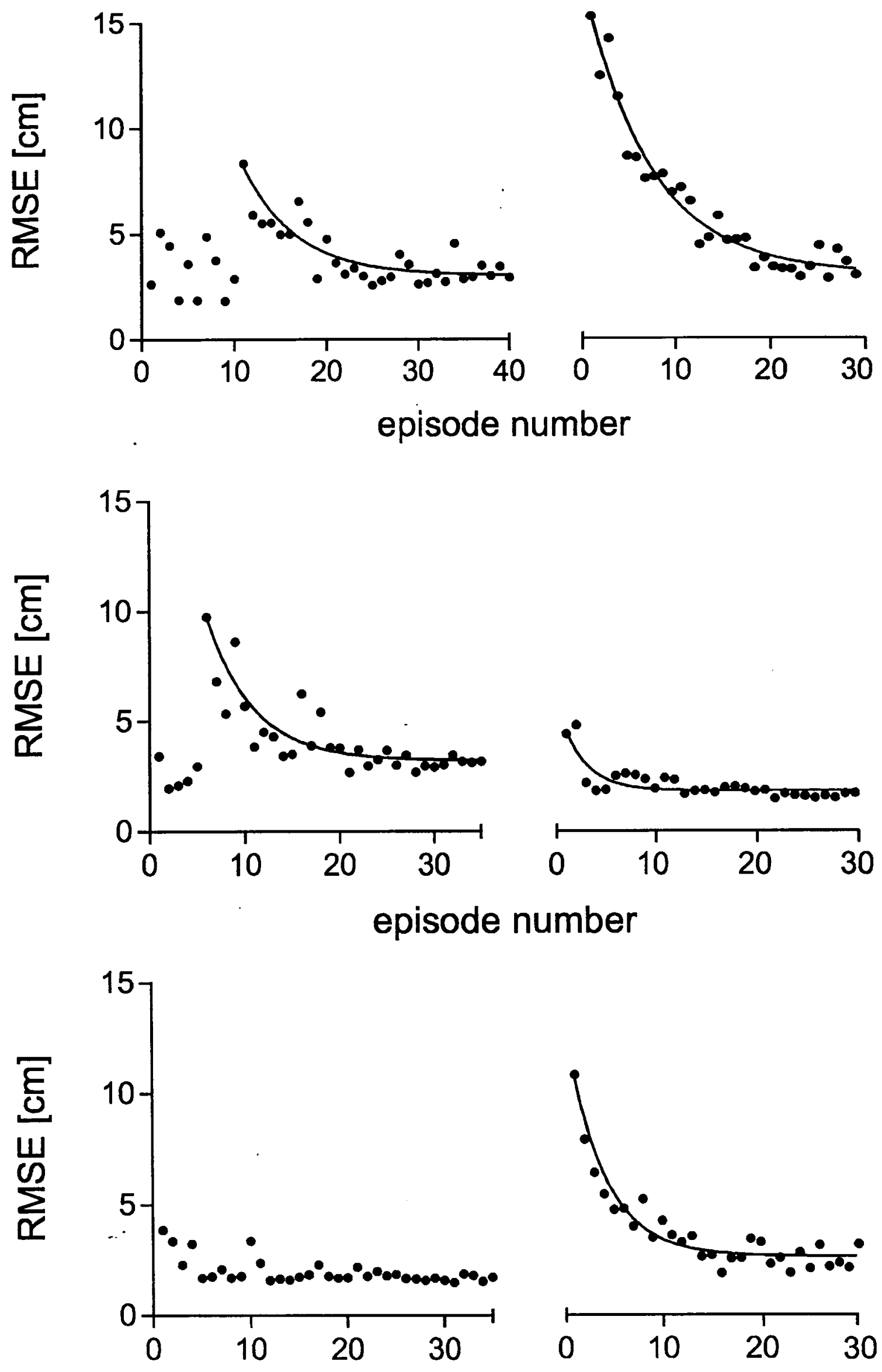
Table 1

\begin{tabular}{clrr}
\hline Experiment & & \multicolumn{1}{c}{ session 1 } & \multicolumn{1}{c}{ session 2 } \\
\hline \multirow{3}{*}{$\mathrm{B}$} & initial error & $11.59+/-3.32$ & $17.74+/-4.29$ \\
& final error & $3.78+/-0.93$ & $4.67+/-1.54$ \\
& half-time & $4.21+/-1.56$ & $5.93+/-2.38$ \\
\hline \multirow{3}{*}{$\mathrm{C}$} & initial error & $11.29+/-2.58$ & $8.23+/-3.80$ \\
& final error & $3.20+/-1.10$ & $2.58+/-0.69$ \\
& half-time & $3.80+/-2.02$ & $2.29+/-1.52$ \\
\hline \multirow{3}{*}{$\mathrm{D}$} & initial error & $\mathrm{N} / \mathrm{A}$ & $11.91+/-3.74$ \\
& final error & $\mathrm{N} / \mathrm{A}$ & $2.72+/-0.56$ \\
& half-time & $\mathrm{N} / \mathrm{A}$ & $2.01+/-1.29$ \\
\hline
\end{tabular}

Table 2

\begin{tabular}{|c|c|c|c|c|}
\hline Experiment & & Session & Pause Length & $\mathrm{S} * \mathrm{P}$ \\
\hline \multirow[t]{3}{*}{ 工 } & initial error & $95.65^{\text {*** }}$ & $0.54^{\mathrm{ns}}$ & $1.96^{\mathrm{ns}}$ \\
\hline & final error & $21.85^{* * *}$ & $1.66^{\mathrm{ns}}$ & $1.45^{\mathrm{ns}}$ \\
\hline & half-time & $17.84^{* * *}$ & $0.79^{\mathrm{ns}}$ & $1.35^{\mathrm{ns}}$ \\
\hline \multirow{3}{*}{$\mathrm{C}$} & initial error & $9.33^{*}$ & $1.46^{\mathrm{ns}}$ & $0.79^{\text {ns }}$ \\
\hline & final error & $11.62^{* *}$ & $0.68^{\text {ns }}$ & $2.51^{\mathrm{ns}}$ \\
\hline & half-time & $7.11^{*}$ & $0.38^{\text {ns }}$ & $1.22^{\mathrm{ns}}$ \\
\hline
\end{tabular}

